This is the author's final, peer-reviewed manuscript as accepted for publication. The publisher-formatted version may be available through the publisher's web site or your institution's library.

\title{
An inverse problem for the heat equation II
}
A. G. Ramm

\section{How to cite this manuscript}

If you make reference to this version of the manuscript, use the following information:

Ramm, A. G. (2002). An inverse problem for the heat equation II. Retrieved from http://krex.ksu.edu

\section{Published Version Information}

Citation: Ramm, A. G. (2002). An inverse problem for the heat equation II. Applicable Analysis: An International Journal, 81(4), 929-937.

Copyright: $\odot 2002$ Taylor \& Francis Ltd

Digital Object Identifier (DOI): doi:10.1080/0003681021000004519

Publisher's Link: http://www.tandfonline.com/doi/abs/10.1080/0003681021000004519

This item was retrieved from the K-State Research Exchange (K-REx), the institutional repository of Kansas State University. K-REx is available at http://krex.ksu.edu 
Applic. Analysis, 81, N4, (2002), 929-937. 


\title{
An inverse problem for the heat equation II *广
}

\author{
A. G. Ramm \\ LMA/CNRS, 31 Chemin J.Aiguier \\ Marseille 13402, France \\ and Department of Mathematics, \\ Kansas State University \\ Manhattan, Kansas, 66506-2602, USA \\ email: ramm@math.ksu.edu
}

\begin{abstract}
Completeness of the set of products of the derivatives of the solutions to the equation $\left(a v^{\prime}\right)^{\prime}-\lambda v=0, v(0, \lambda)=0$ is proved. This property is used to prove the uniqueness of the solution to an inverse problem of finding conductivity in the heat equation $\dot{u}=\left(a(x) u^{\prime}\right)^{\prime}, u(x, 0)=0, u(0, t)=$ $0, u(1, t)=f(t)$ known for all $t>0$, from the heat flux $a(1) u^{\prime}(1, t)=g(t)$. Uniqueness of the solution to this problem is proved. The proof is based on Property C. It is proved the inverse that the inverse problem with the extra data (the flux) measured at the point, where the temperature is kept at zero, (point $x=0$ in our case) does not have a unique solution, in general.
\end{abstract}

\section{Introduction}

In a study of one and multidimensional inverse problems Property $\mathrm{C}$ (completeness of the set of products of solutions to some homogeneous equations) was introduced and has been used extensively [3],[5]. Completeness of the set of products of the eigenfunctions of some Sturm- Liouville equations was studied in [1], and, in a more general context, in [3]-[6]. In this paper we prove completeness of the set of products of the derivatives of solutions to a second-order equation and use it to study an inverse problem for it. A similar idea was used in [5] in the multidimensional case. It is of interest to see that property $\mathrm{C}$ allows one to decide which measurements are most informative: we prove, using this property, that the measurements of the heat flux at the point $x=1$ allow one to uniquely determine the conductivity, while the measurements of the heat flux at the point $x=0$ do not allow one to determine the conductivity uniquely, in general.

*Primary: 35R30, 81U40; Secondary: 47A40

†inverse problem, conductivity, flux, property C, potential, Sturm- Liouville equations 
Denote $\dot{u}:=\frac{\partial u}{\partial t}, u^{\prime}:=\frac{\partial u}{\partial x}$. Consider the following problem:

$$
\begin{gathered}
\dot{u}=\left(a(x) u^{\prime}\right)^{\prime}, 0 \leq x \leq 1, t>0, \\
u(x, 0)=0, \\
u(0, t)=0, u(1, t)=f(t), \\
a(1) u^{\prime}(1, t)=g(t) .
\end{gathered}
$$

Assume:

$$
f \in L^{1}([0,1]), f \not \equiv 0, a \in W^{2,1}([0,1]), a>0,
$$

where $W^{2,1}$ is the Sobolev space of functions twice differentiable in $L^{1}([0,1])$. The function $u(x, t)$ is the temperature of a medium, $a(x)$ is its conductivity, $g(t)$ is the heat flux (through the point $x=1$ ), which can be measured experimentally. The inverse problem is to find the conductivity of the medium from the flux measurements. Formulas for finding conductivity at the boundary in the multidimensional case were derived in [6].

Applying the Laplace transform to (1.1)-(1.3) one gets the equation $\left(a(x) v^{\prime}\right)^{\prime}-$ $\lambda v=0$ ( $\lambda$ is the spectral parameter $)$ with certain boundary conditions and extra data. Our uniqueness result for (1.1)-(1.4) follows from the completeness of the set of products of the derivatives of the solutions to this equation. It is interesting to note that if (1.4) is replaced by

$$
a(0) u^{\prime}(0, t)=h(t)
$$

the result is no longer valid and, in fact, only 'one half' of $a(x)$ can be recovered, in general. Similar non-uniqueness result was obtained in [7].

The structure of this paper is the following. In section 2 we reduce the completeness of the set of products of the derivatives of the solutions to Property $\mathrm{C}$ for a Sturm-Liouville equation with the third boundary condition. Though Property $\mathrm{C}$ for this equation follows from a result in [1], we give a simple alternative proof. In section 3 we use Property $\mathrm{C}$ to prove the uniqueness result for (1.1)-(1.4) and also a non-uniqueness for (1.1)-(1.3),(1.5).

\section{Completeness of the set of products of the derivatives of the solutions}

Theorem 2.1. Let $v_{i}, i=1,2$, solve the equations

$$
\left(a_{i}(x) v_{i}^{\prime}\right)^{\prime}-\lambda v_{i}=0,0 \leq x \leq 1, v_{i}(0, \lambda)=0,
$$

where $0<a_{i} \in W^{1,2}([0,1])$. Then the set of products $\left\{v_{1}^{\prime}(\cdot, \lambda) v_{2}^{\prime}(\cdot, \lambda) \mid \forall \lambda>0\right\}$ is complete in $L^{1}([0,1])$.

To prove this theorem we need some lemmas. 
Lemma 2.1. A function $w$ solves the problem

$$
w^{\prime \prime}-\lambda a^{-1}(x) w=0, w^{\prime}(0, \lambda)=0
$$

if and only if $v(x):=\int_{0}^{x} a^{-1}(t) w(t) d t$ solves the problem

$$
\left(a v^{\prime}\right)^{\prime}-\lambda v=0, v(0, \lambda)=0 .
$$

Proof. Let $v$ satisfy $(2.3)$ and let $w:=a v^{\prime}$. Then $v(x):=\int_{0}^{x} a^{-1}(t) w(t) d t$, and a differentiation of (2.3) yields (2.2).

Conversely, let $w$ solve $(2.2)$, and define $v(x):=\int_{0}^{x} a^{-1}(t) w(t) d t$. Integrating (2.2) from 0 to $x$ one gets $\left(a v^{\prime}\right)^{\prime}(x)-\lambda v(x)=0$ and $v(0)=0$.

By Lemma 2.1 the set of the derivatives of the solutions to (2.3) coincides, up to a factor $a^{-1}(x)$, with the set of the solutions to (2.2). Our next step is to reduce (2.2) to a Sturm-Liouville equation.

Lemma 2.2. Under the substitution $\xi(x):=\int_{0}^{x} a^{-1 / 2}(t) d t, w(x)=a^{1 / 4}(x) z(\xi(x))$ the problem (2.2) is equivalent to the problem:

$$
\ddot{z}-q(\xi) z-\lambda z=0, \dot{z}(0)+h z(0)=0,
$$

where $\dot{z}:=\frac{d z}{d \xi}, q(\xi(x)):=\frac{3}{16} a^{-1}(x)\left[a^{\prime}(x)\right]^{2}-\frac{1}{4} a^{\prime \prime}(x), h:=\frac{1}{4} a^{-1 / 2}(0) a^{\prime}(0)$.

Proof. Make the following substitutions

$$
\xi(x):=\int_{0}^{x} \phi(t) d t, w(x):=z(\xi(x)) \psi(x),
$$

where the functions $\phi$ and $\psi$ are to be determined. Let us derive an equation for $z$ assuming that $w$ satisfies (2.2). One has

$$
\begin{gathered}
w^{\prime}=\psi^{\prime} z+\psi \phi \dot{z}, \\
w^{\prime \prime}=\psi^{\prime \prime} z+\psi^{\prime} \phi \dot{z}+\psi^{\prime} \phi \dot{z}+\psi \phi^{\prime} \dot{z}+\psi \phi^{2} \ddot{z}=\psi^{\prime \prime} z+\left(2 \psi^{\prime} \phi+\psi \phi^{\prime}\right) \dot{z}+\psi \phi^{2} \ddot{z} .
\end{gathered}
$$

Thus,

$$
w^{\prime \prime}-\lambda a^{-1} w=\psi \phi^{2}\left(\ddot{z}+\frac{2 \psi^{\prime} \phi+\psi \phi^{\prime}}{\psi \phi^{2}}+\frac{\psi^{\prime \prime}}{\psi \phi^{2}} z-\lambda \frac{a^{-1} \psi}{\psi \phi^{2}} z\right)=0 .
$$

Now we choose $\phi=a^{-1 / 2}$ and $\psi:=a^{1 / 4}$ so that $2 \psi^{\prime} \phi+\psi \phi^{\prime}=0$ and $\psi \phi^{2}=a^{-1} \psi$. Then

$$
\ddot{z}+\frac{\psi^{\prime \prime}}{\psi \phi^{2}} z-\lambda z=\ddot{z}+\left(a^{1 / 4}\right)^{\prime \prime} a^{3 / 4} z-\lambda z=\ddot{z}-\left[\frac{3}{16} a^{-1}\left(a^{\prime}\right)^{2}-\frac{1}{4} a^{\prime \prime}\right] z-\lambda z=0 .
$$


The boundary condition is

$$
w^{\prime}(0)=\left(\frac{1}{4} a^{-3 / 4} a^{\prime} z+a^{-1 / 4} \dot{z}\right)(0)=a^{-1 / 4}(0)\left[\dot{z}(0)+\frac{1}{4} a^{-1 / 2}(0) a^{\prime}(0)\right]=0 .
$$

Since these transformations are invertible, one concludes that $z$ satisfies (2.4) if and only if $w$ satisfies $(2.2)$.

Lemma 2.3. Consider two equations of the type (2.4) with $q=q_{i}$ and $h=h_{i}$, where $i=1,2$. Then the set of products of the solutions $\left\{z_{1}(\cdot, \lambda) z_{2}(\cdot, \lambda) \mid \forall \lambda>0\right\}$ is dense in $L^{1}([0,1])$.

Proof. Let $z_{0}$ be a solution to (2.4) with $q=0$. Then, up to a multiplicative constant, one has: $z_{0}(\xi)=\cos (k \xi)+\frac{h}{k} \sin (k \xi), \lambda=-k^{2}$. For $z_{1}$ and $z_{2}$ one has a representation through the transformation kernels $K_{1}, K_{2}$, which depend on $q_{i}$ and $h_{i}$ :

$$
z_{i}(\xi, \lambda)=z_{0}(\xi, \lambda)+\int_{0}^{\xi} K_{i}(\xi, \eta) z_{0}(\eta, \lambda) d \eta .
$$

The properties of the transformation operators can be found, e.g., in [1] and [5]. Also

$$
z_{0}(\xi, \lambda)=\cos (k \xi)+h \int_{0}^{\xi} \cos (k s) d s
$$

Therefore,

$$
z_{i}(\xi, \lambda)=\cos (k \xi)+\int_{0}^{\xi} T_{i}(\xi, \eta) \cos (k \eta) d \eta
$$

where

$$
T_{i}(\xi, \eta)=h_{i}+K_{i}(\xi, \eta)+h_{i} \int_{0}^{\xi} K_{i}(\xi, s) d s .
$$

We have used above the following formula

$$
\int_{0}^{\xi} K_{i}(\xi, \eta) \int_{0}^{\eta} \cos (k s) d s d \eta=\int_{0}^{\xi} \cos (k \eta)\left(\int_{\eta}^{\xi} K_{i}(\xi, s) d s\right) d \eta
$$

Completeness of the set of products of the functions of the form (2.5) was proved in [4].

Proof of theorem 2.1. Consider the set of products $w_{1}(\cdot, \lambda) w_{2}(\cdot, \lambda)$, where $w_{i}$ are the solutions to $(2.2)$ with $a=a_{i}$. By Lemma 2.2

$$
w_{1}(x, \lambda) w_{2}(x, \lambda)=\left(a_{1}(x) a_{2}(x)\right)^{1 / 4} z_{1}\left(\xi_{1}(x), \lambda\right) z_{2}\left(\xi_{2}(x), \lambda\right) .
$$


Define the linear operator $(A f)(x):=\left(a_{1}(x) a_{2}(x)\right)^{1 / 4} f(x)$. This is a bounded linear operator on $L^{1}([0,1])$, which has a bounded inverse since $a_{i}$ are continuous and positive. Also $A\left(z_{1} z_{2}\right)=w_{1} w_{2}$ and the same is true for linear combinations of the products. Since these combinations are dense in $L^{1}([0, \xi(1)]$ by Lemma 2.3 and $A$ is a linear isomorphism we conclude that linear combinations of products of $w_{i}$ are dense in $L^{1}([0,1])$. By Lemma $(2.1) w_{1} w_{2}=v_{1}^{\prime} v_{2}^{\prime}$, where $v_{i}$ are solutions to 2.1 and the system of products of the derivatives is, therefore, complete.

\section{Finding conductivity: uniqueness and non- uniqueness}

Theorem 3.1. There exists at most one solution to the inverse problem (1.1)(1.4).

Proof. Define $v(x, \lambda):=\int_{0}^{\infty} e^{-\lambda t} u(x, t) d t$. Applying the Laplace transform to equations (1.1)-(1.4) one gets

$$
\begin{gathered}
\left(a(x) v^{\prime}\right)^{\prime}-\lambda v=0,0 \leq x \leq 1, \lambda>0, \\
v(0, \lambda)=0, v(1, \lambda)=F(\lambda):=\int_{0}^{\infty} e^{-\lambda t} f(t) d t, \\
a(1) v^{\prime}(1, \lambda)=G(\lambda):=\int_{0}^{\infty} e^{-\lambda t} g(t) d t .
\end{gathered}
$$

Assume that there exist $a_{1}$ and $a_{2}$ which generate the same data $f$ and $g$ and, therefore, the same $F$ and $G$. Put in equation (3.1) $a=a_{i}, v=v_{i}, i=1,2$, and subtract the second equation from the first to get

$$
\left(a_{1}(x) w^{\prime}\right)^{\prime}-\lambda w=-\left(p(x) v_{2}^{\prime}\right)^{\prime}
$$

where $p:=a_{1}-a_{2}, w:=v_{1}-v_{2}$. Let $\psi$ be an arbitrary solution to the problem:

$$
\left(a_{1}(x) \psi^{\prime}\right)^{\prime}-\lambda \psi=0, \psi(0, \lambda)=0
$$

Multiply (3.4) by $\psi$ and integrate by parts over $[0,1]$ to get:

$$
-\int_{0}^{1} a_{1} w^{\prime} \psi^{\prime} d x+\left.a_{1} w^{\prime} \psi\right|_{0} ^{1}-\lambda \int_{0}^{1} w \psi d x=-\int_{0}^{1} p v_{2}^{\prime} \psi^{\prime} d x+\left.p v_{2}^{\prime} w\right|_{0} ^{1} .
$$

Integrating the first term by parts once more and observing that the non-integral terms vanish because for $x=0$ and $x=1 w(x, \lambda)=0$ by $(3.2), \psi(0)=0$, and 
$a_{1}(1) v_{1}^{\prime}(x, \lambda)=a_{2} v_{2}^{\prime}(1, \lambda)$ by $(3.3)$, one obtains an orthogonality relation:

$$
0=\int_{0}^{1} p v_{2}^{\prime} \psi^{\prime} d x \quad \forall \lambda>0 .
$$

By Theorem 2.1 the set of products $\left\{v_{2}^{\prime}(\cdot, \lambda) \psi^{\prime}(\cdot, \lambda)\right\}$ is complete in $L^{1}(0,1)$. Therefore, $p=0$ and $a_{1}=a_{2}$.

As we have mentioned in the Introduction, the uniqueness of the solution to the inverse problem fails to hold if the flux measurements are taken at the left end of the interval.

Theorem 3.2. Consider problem (1.1)-(1.3) with extra data (1.5). Then there are conductivities $a_{1}$ and $a_{2} \neq a_{1}$ which produce the same flux $h$.

Proof. Laplace transform turns problem (1.1)-(1.3) into (3.1)-(3.2) with the extra data

$$
a(0) v^{\prime}(0, \lambda)=H(\lambda):=\int_{0}^{\infty} e^{-\lambda t} h(t) d t .
$$

Make the substitution $b:=a v^{\prime}$ and write the problem in the form

$$
\begin{gathered}
b^{\prime \prime}-\lambda a^{-1}(x) b=0, \\
b^{\prime}(0, \lambda)=0, \quad b^{\prime}(1, \lambda)=\lambda F(\lambda), \quad b(0, \lambda)=H(\lambda) .
\end{gathered}
$$

Introduce $\theta(x, \lambda)$ as the solution to the problem

$$
\theta^{\prime \prime}-\lambda a^{-1}(x) \theta=0, \quad \theta^{\prime}(0, \lambda)=0, \quad \theta(0, \lambda)=1 .
$$

Clearly, $b(x, \lambda)=c(\lambda) \theta(0, \lambda)$ and, on the one hand,

$$
H(\lambda)=b(0, \lambda)=c(\lambda) \theta(0, \lambda)=c(\lambda),
$$

while on the other hand,

$$
\lambda F(\lambda)=b^{\prime}(1, \lambda)=c(\lambda) \theta^{\prime}(1, \lambda)=H(\lambda) \theta^{\prime}(1, \lambda) .
$$

Thus,

$$
H(\lambda)=\frac{\lambda F(\lambda)}{\theta^{\prime}(1, \lambda)} .
$$

The function $\theta^{\prime}(1, \lambda)$ is an entire function of exponential type, which is determined by its zeros $\lambda_{j}$ by the Hadamard formula. The relation $\theta^{\prime}\left(1, \lambda_{j}\right)=0$ together with (3.7) imply that $\theta\left(x, \lambda_{j}\right)$ are the eigenfunctions of the Neumann problem for equation (3.7) and $\lambda_{j}$ are the corresponding eigenvalues. If some $\phi(x)$ satisfies differential equation (3.7) and the Neumann boundary conditions then $\phi(1-x)$ satisfies equation (3.7) with $a(x)$ replaced by $a(1-x)$ and the Neumann boundary conditions. In other words, the set $\left\{\lambda_{j}\right\}$ will be the same for $a(x)$ and $a(1-x)$. Since the set $\left\{\lambda_{j}\right\}$ determines $\theta^{\prime}(1, \lambda)$ uniquely, this function is the same for two different coefficients $a$. But then by (3.8) and (3.6) the corresponding functions $h$ are also the same. 
Thus, if measurements are taken on a wrong end of the interval, the unique recovery of the conductivity is impossible, in general.

Remark 3.3. The situation is similar to the one for the Sturm-Liouville equation:

$$
v^{\prime \prime}-q(x) v+\lambda v=0, v(0, \lambda)=0, v(1, \lambda)=F(\lambda),
$$

and the extra data $v^{\prime}(0, \lambda)=G(\lambda)$. In this case $q(x)$ and $q(1-x)$ will also produce the same extra data $G$. Even more can be said in this case: $q$ is determined by $G$ on $[0,1 / 2$ ). This follows from the old result in [1] (see also $[2]): q$ on the half of the interval can be recovered from the knowledge of one spectrum for problem (3.9) with Dirichlet boundary conditions. It also follows from Theorem 3.1 in [3].

\section{References}

[1] B.M. Levitan, Generalized translation operators and some of their applications, Jerusalem, Israel Program for Scientific Translations, 1964.

[2] H.Hochshtadt, B.Lieberman, An inverse Sturm-Liouville problem with mixed data, SIAM J.Appl.Math, 34 (1978), 331-348.

[3] A.G.Ramm, Property $C$ for ODE and applications to inverse problems, in the book Operator Theory and Its Applications, Amer. Math. Soc., Fields Institute Communications vol. 25 (2000), pp.15-75, Providence, RI.

[4] A.G.Ramm, Property $C$ for $O D E$ and applications to inverse scattering, Zeit. fuer Angew. Analysis, 18, no.2 (1999), 331-348.

[5] A.G.Ramm, Multidimensional inverse scattering problems, Longman/Wiley, New York, 1992, 1-385.

[6] A.G.Ramm, Finding conductivity from boundary measurements, Comp.\& Math.with Appl., 21, no.8 (1991), 85-91.

[7] A.G.Ramm, An inverse problem for the heat equation Jour. of Math. Anal. Appl., 264, N 2, (2001), 691-697. 